\title{
AVALIAÇÃO DA FLEBOGRAFIA ORBITÁRIA EM OITO CASOS DE SÍNDROME DE TOLOSA-HUNT
}

\author{
D.R. NALLI*,S.M.F. MALHEIROS**, R.G. NOGUEIRA ${ }^{* * * *}$, N. ABDALA*, J.G.P. CALDAS***, \\ H. CARRETE JR. *, A.R. MASSARO**
}

\begin{abstract}
RESUMO - A Síndrome de Tolosa-Hunt (STH) ou oftalmoplegia dolorosa é associada a granulomatose inespecifica de etiologia desconhecida que acomete a fissura orbitária superior. Compromete estruturas nervosas e vasculares causando quadro clínico variável que sempre se associa a dore apresenta resposta favorável à corticoterapia. Processos inflamatórios, tumores e aneurismas dessa região podem causar sintomas semelhantes. A tomografia computadorizada, a angiografia cerebral e a flebografia orbitária são métodos de imagem indicados para orientar o diagnóstico. Revisamos os resultados destes exames radiológicos de oito pacientes atendidos no Hospital São Paulo no período 1989 a 1991, com diagnóstico de STH segundo os critérios de Hunt e Hannerz. A análise das alterações da flebografia orbitária, de acordo com a sistematização feita por Hannerze col. mostrou ser este exame inespecífico, porém capaz de orientar melhor o diagnóstico.
\end{abstract}

PALAVRAS-CHAVE: síndrome de Tolosa-Hunt, oftalmoplegia dolorosa, flebografia orbitária, veia oftálmica superior, seio cavernoso.

\section{Orbital phlebography evaluation in eight cases of Tolosa-Hunt syndrome}

SUMMARY - Tolosa-Hunt syndrome (THS), or painful ophthalmoplegia is associated to a non-specific granulomatosis of unknown etiology, that involves the superior orbital fissure and its nervous and vascular structures. The clinical picture that responds to steroid therapy, is variable and is always associated with pain. Inflammatory conditions, tumors and aneurysms can produce similar symptoms. Computed tomography, cerebral angiography and ortital phlebography are the imaging methods of choice for making the diagnosis. We revised the results of these radiological examinations of eight patients seen at the Hospital São Paulo from 1989 to 1991, with the diagnosis of THS according to Hunt and Hannerz criteria. The analysis of orbital phlebographic changes based upon Hannerz et al. systematization showed non-specific features, but those were able to help the diagnosis.

KEY WORDS: Tolosa-Hunt, painful ophthalmoplegia, orbital phlebography, superior ophthalmic vein, cavernous sinus.

A síndrome de Tolosa Hunt (STH), também conhecida como oftalmoplegia dolorosa, é associada a uma granulomatose inespecífica de etiologia desconhecida que acomete a região da fissura orbitária superior e do seio cavernoso. O quadro clínico consiste de dor periorbitária associada ou não a dor hemicrânica, paralisias de intensidade variável da musculatura ocular extrínseca homolateral, concomitantemente podendo ocorrer comprometimento do sistema simpático ocular e alterações sensitivas nos territórios oftálmico e maxilar do trigêmeo. A terapêutica com corticoesteróides provoca rápida regressão dos sintomas, principalmente da dor, tornando-se importante elemento diagnóstico. O diagnóstico diferencial deve ser estabelecido com outras causas que provocam sintomas semelhantes, entre elas aneurismas, tumores para-selares e processos

Hospital São Paulo, Escola Paulista de Medicina (EPM):*Pós-Graduando de Neurorradiologia, EPM; **Mestre em Neurologia EPM; ***Mestre em Neurorradiologia, EPM; ****Prof. Assistente de Neurorradiologia EPM. Aceite: 12-outubro-1993.

Dr. Roberto Gomes Nogueira - Departamento de Diagnóstico por Imagem (DDI), Escola Paulista de Medicina - Rua Botucatu 740 - 04023-900 São Paulo SP - Brasil. 
inflamatórios envolvendo o seio cavernoso ou fissura orbitária superior. Os métodos de imagem excluem estas causas e podem oferecer subsídios para o diagnóstico da STH. Atualmente são utilizadas a tomografia computadorizada (TC), a angiografia cerebral (AC), a flebografia orbitária (FO) e a ressonância magnética (RM).

Apresentamos uma comparação dos resultados destes métodos na investigação radiológica da STH, excluindo-se a RM que, por diversos motivos, ainda é pouco usada em nosso meio, e analisamos as diversas alterações que ocorrem na FO para avaliar sua importância no diagnóstico da STH.

\section{CASUÍSTICA E MÉTODO}

Foram revisados os exames radiológicos de oito pacientes com diagnóstico de STH, com sintomas cuja evolução variou de 3 a 90 dias, atendidos no Hospital São Paulo no período de 1987 a 1991 (Tabela 1). O diagnóstico de STH foi definido segundo os critérios propostos por $\mathrm{Hunt}^{6,7} \mathrm{e}$ sistematizados por Hannerz e col$^{5}: 1$ ) dor periorbitária ou hemicrânica constante; 2) acometimento parcial ou total de pelo menos um dos nervos oculomotores ipsilaterais; 3) exclusão de outras possíveis causas de síndrome para-selar por exames de radiografia simples do crânio, TC (cortes axiais e coronais sem e com injeção endovenosa de meio de contraste) e AC.

Tabela 1. Distribuição quanto à idade, sexo, evolução e quadro clínico dos pacientes estudados com diagnósticos de SHT.

\begin{tabular}{ccccl}
\hline Caso & Idade (anos) & Sexo & Evolução (dias) & \multicolumn{1}{c}{ Quadro Clínico } \\
\hline \hline 1 & 39 & $\mathrm{M}$ & 3 & $3^{\circ}$ nervo E \\
2 & 31 & $\mathrm{M}$ & 16 & $3^{\circ}$ nervo E \\
3 & 43 & $\mathrm{~F}$ & 90 & $\mathrm{RS}+\mathrm{RM}+\mathrm{RL} \mathrm{E}$ \\
4 & 38 & $\mathrm{M}$ & 15 & $\mathrm{RS}+$ Elevador da pálpebra E \\
5 & 49 & $\mathrm{M}$ & 25 & $3^{\circ}$ incompleto $+4^{\circ}+6^{\circ}$ nervos E \\
6 & 62 & $\mathrm{M}$ & 20 & $\mathrm{RL}+\mathrm{RM}+$ ptose palpebral D \\
7 & 27 & $\mathrm{M}$ & 60 & $3^{\circ}$ incompleto + $6^{\circ}$ nervos D \\
8 & 66 & $\mathrm{M}$ & 15 & $3^{\circ}$ nervo incompleto E \\
\hline
\end{tabular}

M, masculino; F, feminino; $R S$, músculo reto superior; $R L$, músculo reto lateral; $R M$, músculo reto medial.

Todos foram submetidos a FO, cujas imagens foram avaliadas por método duplo cego por observador experiente. As FO foram realizadas de acordo com a técnica descrita por Vritsos ${ }^{22} \mathrm{e}$ modificada por Vignaud e $\mathrm{col}^{21}$. Esta técnica consiste na punção de veia frontal superficial com agulha calibre $21 \mathrm{G}$, injetando-se $8 \mathrm{ml} \mathrm{de}$ meio de contraste iodado hidrossolúvel, em aproximadamente 2 segundos. Previamente à injeção, fazse tomiquete ao redor do crânio, ajustando-o ao nível do lábio superior, para compressão das veias angulares e temporais, evitando-se a fuga do meio de contraste para veias superficiais. As radiografias são obtidas durante a injeção dos últimos $2 \mathrm{ml}$ do contraste, nas incidências póstero-anterior, semi-axial e perfil. A incidência em perfil deve ser feita com pequena rotação lateral da cabeça, para evitar a superposição das veias orbitárias superiores.

\section{RESULTADOS}

A AC foi normal em todos os casos. A TC mostrou assimetria do seio cavernoso, nitidamente aumentado no lado acometido em 2 casos (Tabela 2). As FO foram
Tabela 2. Distribuição dos resultados anormais nos diferentes métodos de imagem em relação ao lado acometido.

\begin{tabular}{ccccc}
\hline Caso & TC & AC & FO & Sintoma \\
\hline 1 & N & N & A/E & E \\
2 & N & N & A/E & E \\
3 & N & N & A/E & E \\
4 & N & N & A/E & E \\
5 & A/E & N & A/E & E \\
6 & N & N & A/D & D \\
7 & A/D & N & A/D & D \\
8 & N & N & A/E & E \\
\hline
\end{tabular}

$\mathrm{N}$, normal; A, anormal; E, esquerdo; D, direito. 


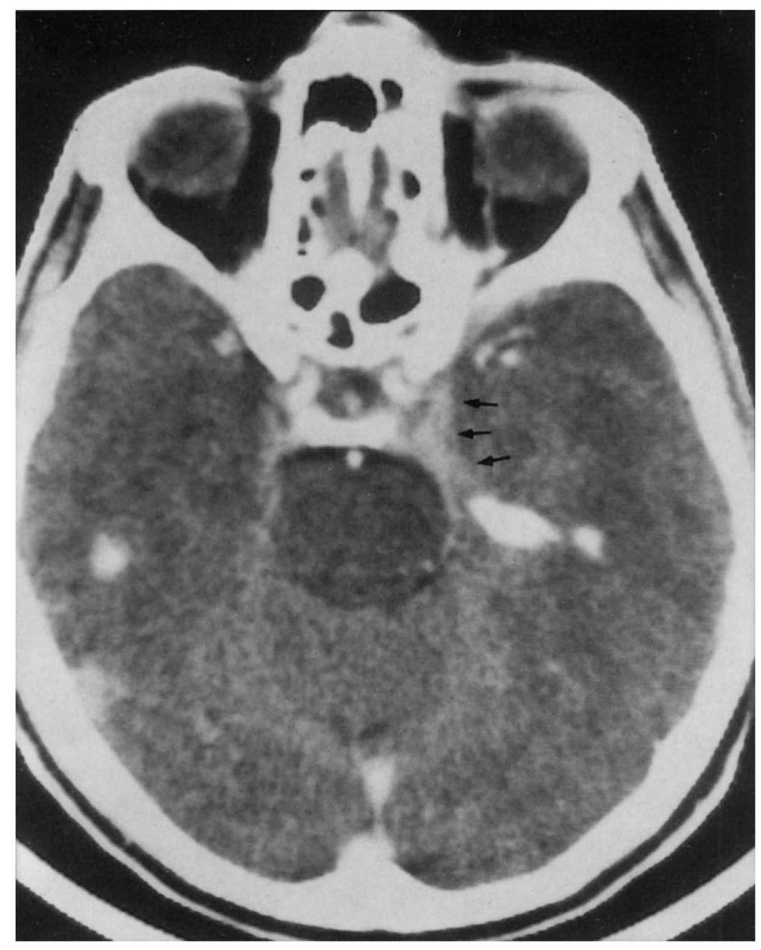

Fig 1. Caso 5. Corte axial de TC de crânio com contraste, evidenciando aumento da área de realce para-selar do lado acometido, com contorno lateral côncavo (setas). anormais em todos os casos. As alteraçōes encontradas nas FO foram divididas em quatro grupos, de acordo com os criterios estabelecidos por Hannerz e $\mathrm{col}^{5}$ : 1 . estreitamento ou irregularidade da veia oftálmica superior (VOS), 2. oclusão parcial ou total da VOS, 3. oclusão parcial ou total do seio cavernoso (SC), 4. circulação colateral venosa.

A oclusão parcial/total do SC ipsilateral foi observada em todos os casos. As outras anormalidades foram observadas em cinco casos (Tabela 3 ).

\section{COMENTÁRIOS}

Em 1954, Tolosa ${ }^{20}$ descreveu o caso de paciente com dor orbitária, perda visual progressiva, oftalmoplegia total e parestesia no território oftálmico do trigêmeo ipsilaterais. A AC mostrou estreitamento da porção cavernosa da carótida interna e a craniotomia exploradora nada revelou. $O$ paciente faleceu três dias após, por complicações cirúrgicas, e a necrópsia mostrou processo inflamatório inespecífico envolvendo a artéria carótida interna e o seio cavernoso do lado acometido. Em 1961, Hunt e $\operatorname{col}^{6}$ descreveram quadro clínico em seis pacientes, que acreditaram ser semelhante à entidade descrita por Tolosa. Em 1966, Smith e Taxdal ${ }^{17}$, ao descreverem cinco casos, utilisaram pela primeira vez o epônimo "síndrome de Tolosa-Hunt", enfatizando o uso do corticoesteroide como teste terapêutico. Em 1982, Kline ${ }^{8}$ ao fazer a revisão dos 146 casos descritos na literatura inglesa estabeleceu suas bases clínicas e laboratoriais ${ }^{9}$.

O teste terapêutico com corticoesteróides já foi considerado quase patognomônico da STH. Existem, entretanto, relatos de casos de linfomas, meningiomas e tumores de células gigantes da região selar e ou para-selar que responderam por algum tempo a este tratamento, mascarando desta forma o diagnóstico ${ }^{20,21}$.

Tabela 3. Distribuição das anormalidades encontradas na FO.

\begin{tabular}{ccccc}
\hline Caso & $\begin{array}{c}\text { Estreitamento } \\
\text { irregularidade VOS }\end{array}$ & Oclusão VOS & Alteração do SC & Circulaçăo Colateral \\
\hline 1 & + & - & + & + \\
2 & + & + & + & + \\
3 & - & + & + & + \\
4 & + & + & + & + \\
5 & + & + & + & + \\
6 & + & + & + & - \\
7 & - & - & + & - \\
8 & - & + & & + \\
\hline
\end{tabular}




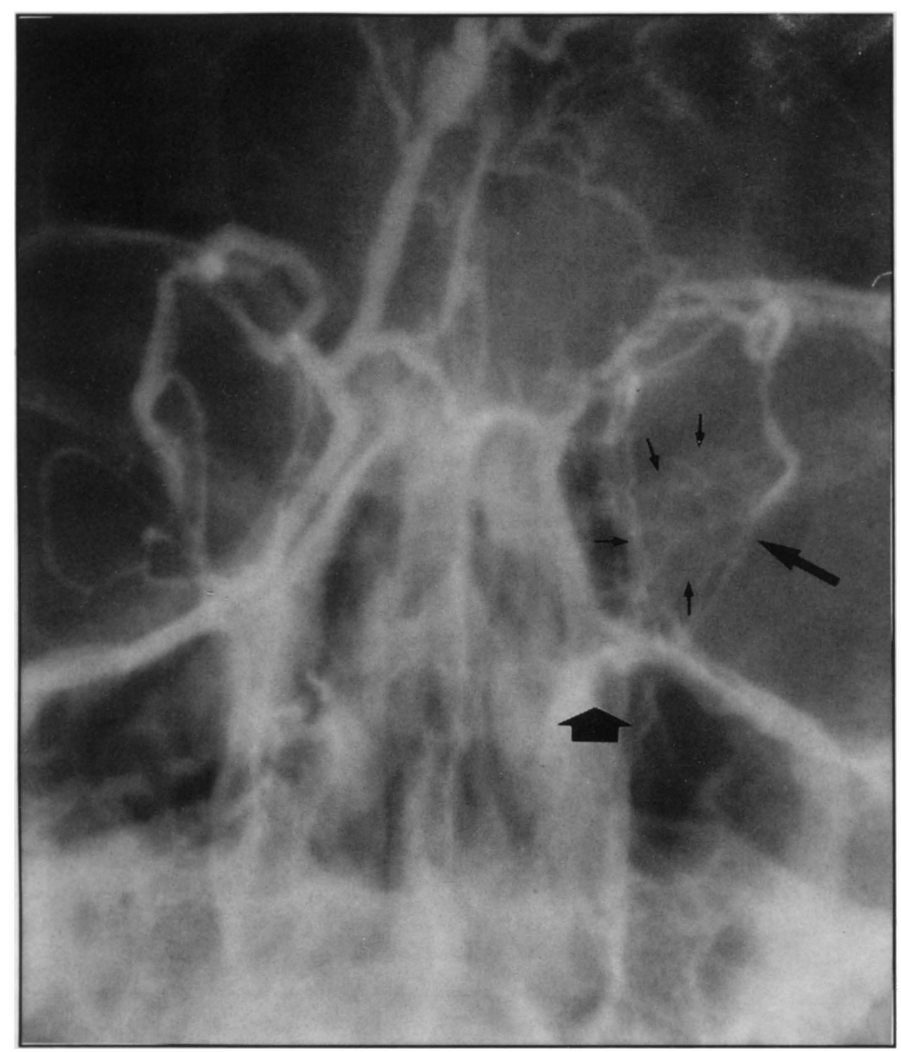

Fig 2. Caso I. Incidência semi-axial da FO, em que se observa estreitamento da porção terminal da VOS (seta longa), irregularidades e má opacificação do seio cavernoso (seta larga), com desenvolvimento de circulação colateral (setas pequenas).

No diagnóstico da STH é sempre necessária a realização da AC, para afastar aneurisma da porção cavernosa da artéria carótida interna. Alguns autores relatam estreitamento segmentar, irregularidade ou constriç̧ão desse segmento arterial no lado acometido e o "fenômeno da onda estacionária", que são alterações de difícil identificação radiológica ${ }^{4,8,11,18}$. A TC ainda é indispensável para o diagnóstico diferencial ${ }^{12,16}$, principalmente nas doenças tumorais para-selares, podendo mostrar alterações sugestivas do envolvimento inflamatório do seio cavernoso e da fissura orbitária superior ${ }^{10,15}$, como foi observado em dois dos nossos casos (Fig 1). O seio cavernoso acometido pela STH geralmente tem contorno lateral côncavo, ao contrário dos neurinomas dessa região, que apresentam contorno lateral convexo. Nos processos inflamatórios, o realce pelo contraste do seio cavernoso é habitualmente pouco intenso, difícultando caracterizar seu envolvimento. Nos aneurismas e meningiomas, ao contrário, o realce é bastante intenso. A ausência do alargamento da veia orbitária superior pode ajudar a estabelecer o diagnóstico diferencial com as fístulas carótidocavernosas e tromboses do seio cavernoso. Estudos recentes descrevem alterações do seio cavernoso na $\mathrm{RM}$, que consistem de imagens com sinais isointensos ao músculo em $\mathrm{T} 1 \mathrm{e}$ isointensos à gordura em T2, com forma e disposição semelhantes às observadas na TC ${ }^{22,23}$.

Milsten e Morretin ${ }^{13}$ descreveram as primeiras flebografias orbitárias para o diagnóstico da STH. Porém, como é comum a assimetria das veias orbitárias superiores e dos seios cavernosos, isto pode dificultar o diagnóstico radiológico'. No entanto, a análise cuidadosa das imagens permite diferenciar as variações anatômicas do comprometimento patológico destas estruturas. Hannerz e col' $^{\text {s }}$ sistematizaram as alterações da FO, classificando-as em quatro grupos: a) estreitamento ou 


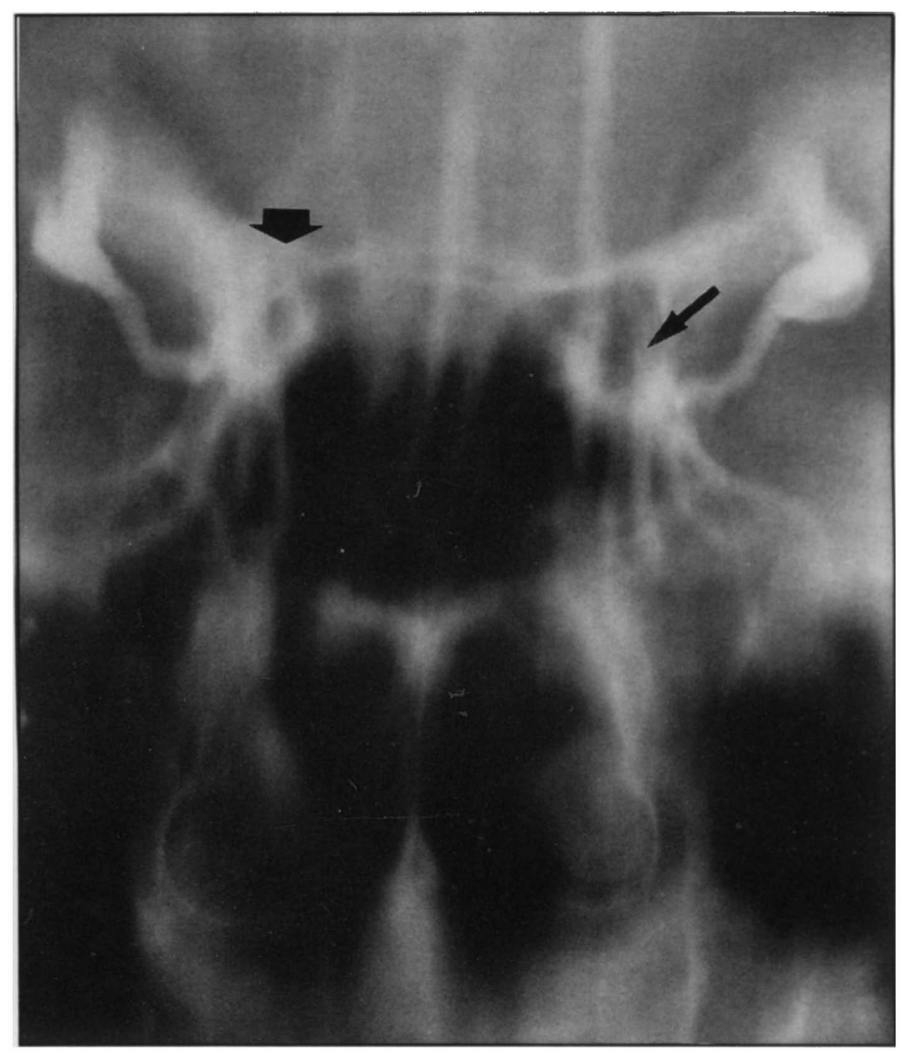

Fig 3. Caso 3. 1\%, em corte planigráfico evidenciando opacificação irregular e incompleta do seio cavernoso esquerdo (seta longa). Aspecto normal da opacificação do seio cavernoso direito (seta larga).

irregularidade da VOS; b) oclusão parcial ou total da VOS; c) oclusão parcial ou total do SC; d) circulação colateral venosa. Compararam 19 pacientes com diagnóstico clínico de STH com 23 normais. Concluiram que, embora sejam frequentes os achados falsos negativos, não existem casos com achados falsos positivos. Encontraram alterações flebográficas em $68 \%$ dos casos de $\mathrm{STH}^{3}$. Muhletaler e col observaram alterações flebográficas em $40 \%$ dos casos de STH por eles estudados ${ }^{14}$.

Em nossa casuística, todas as FO foram anormais e concordantes com o lado acometido, em dois casos (25\%) havendo alteração única (Casos 3 e 8). Nos casos restantes (75\%), observou-se a associação de duas ou mais alterações. Em todos os casos havia alteração (oclusão parcial ou total) do $\mathrm{SC}$ do lado acometido (Figs $2 \mathrm{e} 3$ ). $O$ pequeno número de casos não permite conclusões, porém, não se observou relação entre intensidade do envolvimento neurológico e quantidade de anormalidades encontradas na FO.

Observamos que, quando encontramos associação de algumas das alterações flebográficas sistematizados por Hannerz e col, há maior facilidade na orientação do diagnóstico radiológico de STH. Porém, as alterações na FO não são específicas e podem ser observadas na trombose venosa, flebites e lesões expansivas intra-orbitárias, para-selares ou da fissura orbitária superior.

\section{CONCLUSÃO}

Em nosso estudo, a FO foi o método radiológico que se mostrou alterado em todos os casos e, em nossa opinião, quando as alterações são as sistematizadas por Hannerz e col. e se apresentam associadas, orientam melhor o diagnóstico radiológico da STH No entanto, considerando a 
possibilidade de ser ainda a STH diagnóstico de exclusão, são necessários os exames de AC e TC, para se estabelecer o diagnóstico diferencial de outras doenças com sintomas semelhantes e que apresentam resposta positiva à terapêutica por corticoesteróides.

\section{REFERÊNCIAS}

1. Brismar J. Orbital phlebography: II Anatomy of superior ophthalmic vein and its tributaries. Acta Radiol Diagn 1974, 15: 481-593.

2. Desai SP, Carter J, Jinkins JR. Contrast-enhanced MR imaging of Tolosa-Hunt syndrome: case report. AJNR 1991, 12: 182-183.

3. Fowler TJ, Earl CJ, McAllister VL, McDonald WI. Tolosa-Hunt Syndrome. The dangers of an eponym. Br J Ophthalmol 1975, 59: 149-154.

4. Hallpike JF. Superior orbital fissure syndrome. J Neurosurg Psych 1973, 36: 486-490.

5. Hannerz J, Ericson K, Bergstrand G. Orbital phlebography in patients with Tolosa-Hunt's sydrome in comparison with normal subjects. Acta Radiol Diagn 1984, 25: 457-463.

6. Hunt WE, Meagher JN, Lefever HE, Zeman W. Painful ophthalmoplegia: its relatioship to indolent inflammation of the cavernous sinus. Neurology 1961 11: 56-62.

7. Hunt WE. Tolosa-Hunt syndrome: one cause of painful ophthalmoplegia. J Neurosurg 1976, 44: 544-549.

8. Kettler HL, Martin JD. Arterial stationary wave phenomenon in Tolosa-Hunt syndrome. Neurology 1975, 25: 765-770.

9. Kline LB. The Tolosa-Hunt syndrome. Surv Ophthalmol 1982, 27: 79-95.

10. Kwan ESK, Wolpert SM, Hedges TR III. Tolosa-Hunt revisited : not necessary a diagnosis of exclusion. AJR 1988, 150: 413-418.

11. Mathew NT, Chandy J. Painful ophthalmoplegia. J Neurol Sci 1970, 11: 243-256.

12. McNulty JG - Phlebography of the orbital venous system and the cavernous sinus. Br J Radiol 1969, 42: 113-121.

13. Milstein BA, Morretin LB. Report of a case of sphenoid fissure syndrome studied by orbital venography. Am J Ophthalmol 1971, 72: 600-603.

14. Muhletaler CA, Gerlock AJ. Orbital phlebography in painful ophthalmoplegia (Tolosa-Hunt). Am J Roentgenol 1979, 72: 600-616.

15. Neigel JM, Rootman J, Robinson RG, Durity FA, Nugent RA. The Tolosa-Hunt syndrome: computed tomographic changes and reversal after steroid therapy. Can J Ophthalmol 1986, 21: 287-290.

16. Russel DB, Miller JDR. Orbital venography. Radiology 1972, 103: 267-273.

17. Smith JL, Taxdal DSR. Painful opfthalmoplegia. The Tolosa-Hunt syndrome. Am J Ophthalmol 1966, 61: 1466-1472.

18. Sounheimer FK, Knapp J. Angiographic findings in the Tolosa-Hunt syndrome: painful ophthalmoplegia. Radiology 1973, 106: 105-112.

19. Thomas JE, Yoss RE. The parasellar syndrome: problems in determining etiology. Mayo Clin Proc 1978, 45: 617-623.

20. Tolosa E. Periarteritic lesions of the carotid syphon with clinical features of carotid infraclinoid aneurysm. $J$ Neurol Neurosurg Psychiatry 1954, 17: 300-302.

21. Vignaud J, Clay C, Bilaniuk I. Venography of the orbit: an analytical report of 413 cases. Radiology 1974, 110: 373-381.

22. Vritsos A. A new method for demonstrating ophthalmic veins, facial veins and superficial venous system of the head. Arch Soc Opfthalmol (Grecia) 1961, 12:223-232.

23. Yousem DM, Atlas SW, Grossman RI, Sergot RC, Savino PJ, Bosley TM. MR imaging of Tolosa-Hunt syndrome. AJNR 1989, 10: 1181-1184. 